\title{
Large System Performance of Interference Alignment in Single-Beam MIMO Networks
}

\author{
David A. Schmidt and Wolfgang Utschick \\ Associate Institute for Signal Processing \\ Technische Universität München \\ 80290 Munich, Germany \\ Email: \{dschmidt,utschick\}@tum.de
}

\author{
Michael L. Honig \\ Department of EECS \\ Northwestern University \\ Evanston, Illinois 60208, USA \\ Email: mh@eecs.northwestern.edu
}

\begin{abstract}
We consider a network of $K$ interfering transmitterreceiver pairs, where each node has $N$ antennas and at most one beam is transmitted per user. We investigate the asymptotic performance of different beamforming strategies, as characterized by the slope and y-axis intercept (or offset) of the high signal-tonoise-ratio (SNR) sum rate asymptote. It is known that a slope (or multiplexing gain) of $2 N-1$ is achievable with interference alignment. On the other hand, a strategy achieving a slope of only $N$ might allow for a significantly higher offset. Assuming that the number of fully aligned beamformer sets that achieve a slope of $2 N-1$ is finite for a given channel realization, we approximate the average offset when the best out of a large number $L$ of these sets is selected. We also derive a simple large system approximation for the sum rate of a successive beam allocation scheme when $K=N$. We show that both approximations accurately predict simulated results for moderate system dimensions and characterize the large-system asymptotes for different relationships between $L$ and $N$.
\end{abstract}

\section{INTRODUCTION}

In a MIMO interference network with constant channel coefficients, the optimal high-SNR sum rate scaling (or equivalently, the degrees of freedom, multiplexing gain, or asymptotic slope) is achieved by alignment of the received interference subspaces. That is, the transmit strategies are designed so that the dimension of the subspace spanned by the signals of the interfering transmitters at each receiver is as low as possible [1], [2], [3]. While the asymptotic slope ultimately is the dominant characteristic at very high SNRs, in this work we also examine the $y$-axis intercept (or rate offset) of the high-SNR sum rate asymptote. The rate offset indicates how well the transmit strategies and receive filters, which were designed to remove all interference, match the direct channels between the transmitters and their intended receivers.

We analyze properties of aligned solutions (sets of beamformers and linear receivers) for MIMO interference networks with $K$ users and $N$ antennas at each node (transmitter or receiver). Each user is assumed to transmit a single beam. We focus on the fully aligned scenario with $K=2 N-1$, which achieves the maximum high-SNR sum rate scaling [3]. We start with a small system with $K=3$ and $N=2$ for which there are generally two solutions fulfilling the interference alignment conditions. The solutions can be expressed in closed

This work was supported in part by ARO under grant W911NF-06-1-0339. form and do not depend on the direct channels. For larger systems $(K$ and $N)$ there is in general a finite number of fully aligned solutions. This is because the alignment conditions form a system of polynomial equations where the number of variables matches the number of equations. The exact number of solutions is not known, but is observed to grow very rapidly with $K$ and $N$. Again, the solutions do not depend on the direct channels. All aligned solutions therefore have the same asymptotic slope, but can give different rate offsets.

Exhaustively computing all fully aligned solutions for moderate to large systems is likely to be infeasible. We therefore assume that we can compute a subset such solutions. If we choose the best solution with the largest offset, then the performance clearly depends on how large this subset is. We approximate the performance of this strategy in the large system limit in which $K$ and $N$ tend to infinity with asymptotic ratio $K / N=2$. In this limit it is reasonable to assume that the rate offset follows a Gaussian distribution over the aligned solutions. Taking the aligned solutions with the largest rate offset then corresponds to taking the maximum of a set of samples of the Gaussian random variable. The resulting rate offset can be analyzed via extreme statistics.

When $K<2 N-1$ the set of aligned solutions spans a subspace. Again the objective is to choose an aligned solution in this subspace that best matches the direct channels, and thereby maximizes the rate offset. We compare the performance of fully aligned solutions with the performance of a system in which $K=N$. In that scenario zero interference can be achieved with any set of beams for which the associated received signals are linearly independent. Although the highSNR slope is $N$, as opposed to $2 N-1$ for the fully-aligned scenario, the rate offset can be significantly larger. This is because choosing the beams to be matched to the direct channels gives a much higher sum rate than full alignment at low SNRs. We compute a simple approximation for the rate offset of a particular strategy in which the users' beams are successively optimized. Comparisons with simulations show that this analysis accurately predicts the performance with numerically optimized beams and receivers.

Because of the different rate offsets and slopes for $K=N$ and $K=2 N-1$, the two high-SNR asymptotes intersect at a finite SNR, which depends on how many aligned solutions are 
sampled. The preceding analysis is used to characterize this intersection and shows that when $L$ increases exponentially with $N$, the corresponding SNR increases with system size. Furthermore, for moderate-size systems alignment achieves the larger sum rate for SNRs greater than about $10 \mathrm{~dB}$.

\section{SYSTEM MODEL}

The channel matrix between transmitter $j$ and receiver $k$ is $\boldsymbol{H}_{k j} \in \mathbb{C}^{N \times N}$ and remains constant over time. Each user $k$ is assumed to transmit a single unit variance data stream $b_{k}$, precoded with the beamforming vector $\boldsymbol{v}_{k}$, which is subject to a unit power constraint $\left\|\boldsymbol{v}_{k}\right\|_{2}^{2} \leq 1$. The signal received by user $k$ is

$$
\boldsymbol{y}_{k}=\boldsymbol{H}_{k k} \boldsymbol{v}_{k} b_{k}+\sum_{j \neq k} \boldsymbol{H}_{k j} \boldsymbol{v}_{j} b_{j}+\boldsymbol{n}_{k}
$$

where the additive Gaussian noise $\boldsymbol{n}_{k} \in \mathbb{C}^{N}$ at each receiver has the covariance $\mathrm{E}\left[\boldsymbol{n}_{k} \boldsymbol{n}_{k}^{\mathrm{H}}\right]=\sigma^{2} \mathbf{I}$, i. e., it is uncorrelated over the receive antennas. Receiver $k$ is interested in decoding $b_{k}$ so that the portion of the received signal originating from the transmitters $j \neq k$ is interference.

An expression for the achievable rate for user $k$ is obtained by assuming that all users employ Gaussian codebooks, that the interference is treated as noise, and that the desired signal $b_{k}$ is estimated by means of a linear receive filter $\boldsymbol{g}_{k}$, which we assume w.1.o.g. to have unit norm, i.e., $\left\|\boldsymbol{g}_{k}\right\|_{2}^{2}=1$ :

$$
R_{k}=\log \left(1+\frac{\left|\boldsymbol{g}_{k}^{\mathrm{H}} \boldsymbol{H}_{k k} \boldsymbol{v}_{k}\right|^{2}}{\sum_{j \neq k}\left|\boldsymbol{g}_{k}^{\mathrm{H}} \boldsymbol{H}_{k j} \boldsymbol{v}_{j}\right|^{2}+\sigma^{2}}\right),
$$

in nats per channel use. The achievable sum rate is

$$
R_{\text {sum }}=\sum_{k} R_{k} .
$$

From (2) the summands $R_{k}$ of $R_{\text {sum }}$ for which the interference power term is zero approach $\log \sigma^{-2}$ plus some constant as $\sigma^{-2} \rightarrow \infty$, i.e., for high SNR. The other summands of $R_{\text {sum }}$ approach some constant value as $\sigma^{-2} \rightarrow \infty$. We can therefore write the high-SNR asymptote of $R_{\text {sum }}$ as $s \log \sigma^{-2}+r$, where $s$ is the number of users $k$ for which $\boldsymbol{g}_{k}^{\mathrm{H}} \boldsymbol{H}_{k j} \boldsymbol{v}_{j}=0$ holds for all $j \neq k$. The offset $r$ follows from

$$
r=\lim _{\sigma^{-2} \rightarrow \infty} R_{\text {sum }}-s \log \sigma^{-2} .
$$

We interpret $s$ as the slope and $r$ as the y-axis intercept (or rate offset) of the high-SNR asymptote of the achievable sum rate $R_{\text {sum }}$ plotted versus the SNR in $\mathrm{dB}$, cf. Fig. 1 . The slope $s$ corresponds to the number of users for which the receive filter is able to cancel all interference completely. It is also known as the multiplexing gain or degrees of freedom. If all $K$ users are interference free, then $s=K$ and (4) becomes

$$
r=\sum_{k} \log \left(\left|\boldsymbol{g}_{k}^{\mathrm{H}} \boldsymbol{H}_{k k} \boldsymbol{v}_{k}\right|^{2}\right) .
$$

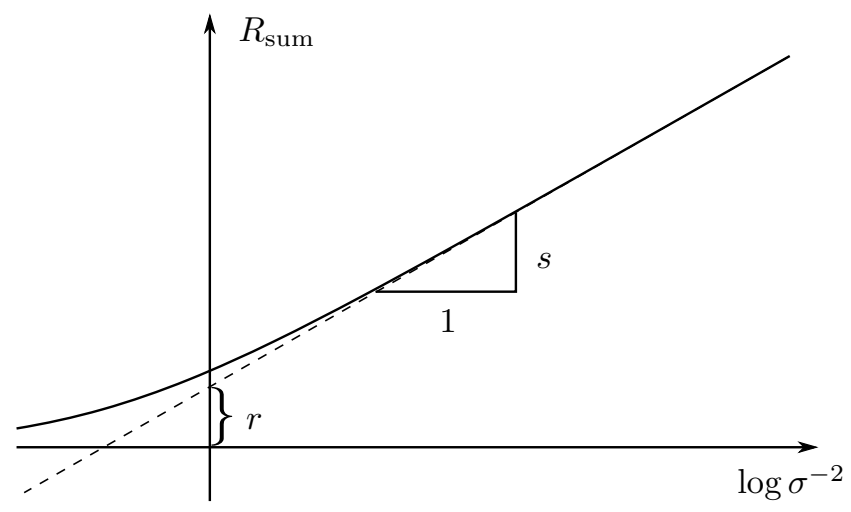

Fig. 1. High-SNR asymptote of achievable sum rate

\section{INTERFERENCE ALIGNMENT}

To maximize the sum rate at high SNR, it is clearly necessary to maximize the slope $s$, i. e., the number of users that can be served without interference. In [2], [3], it is shown that if the channel coefficients are drawn from a continuous distribution, then it is (almost always) possible to find interference free sets $\left\{\boldsymbol{v}_{k}, \boldsymbol{g}_{k}\right\}$ if and only if $K \leq 2 N-1$.

The rationale behind this result is that the zero-interference conditions form a system of multivariate polynomial equations, and that such a system has a solution if the number of equations does not exceed the number of variables unless the equations are inconsistent. When the coefficients of the system of equations (i.e., the cross-channel coefficients) are independent and drawn from a continuous distribution, the probability of getting inconsistent equations is shown to be zero.

One way of viewing this system of equations is that the variables are the real and imaginary parts of the components of all vectors $\boldsymbol{v}_{k}$ and $\boldsymbol{g}_{k}$ (4KN variables); the equations are the unit norm constraints ( $2 K$ quadratic equations), the zero-interference constraints of the form $\boldsymbol{g}_{k}^{\mathrm{H}} \boldsymbol{H}_{k j} \boldsymbol{v}_{j}=0$ $(2 K(K-1)$ real-valued bilinear equations), and another $2 K$ constraints that arbitrarily fix the phase of one component in the beamformers and receive filters to account for the fact that the solutions are invariant to multiplication with $\mathrm{e}^{\mathrm{j} \varphi}$.

Other equivalent ways of expressing the conditions are possible, e.g., with complex variables instead of the real and imaginary parts, or with rank constraints or determinants instead of the bilinear zero-interference constraints. It is common to all, however, that when $K \leq 2 N-1$ holds, the number of variables does not exceed the number of equations.

By the same argument, we will assume that for the fullyaligned scenario with $K=2 N-1$, the set of solutions to the zero-interference (or interference alignment) conditions is discrete [3]. Note that each of these solutions has the same slope $s=2 N-1$, but that they generally differ in the offset $r$.

We also note that the system of equations for alignment does not depend on the direct channel gains $\boldsymbol{H}_{k k}$. Consequently, one possible strategy for maximizing $R_{\text {sum }}$ at high SNR is to 
find all (or as many as possible) solutions to the interference alignment conditions and to select the one that 'matches' the direct channels best, i.e., the one with the highest $r$. In the following, we attempt to approximate the average performance of this strategy assuming a Gaussian i.i.d. channel model, in which the elements of all channel matrices have unit variance.

\section{Three Users And Two Antennas}

To illustrate our analytical approach, which we will subsequently extend to large systems, we first examine the smallest non-trivial scenario, where all terminals have $N=2$ antennas. According to the preceding discussion, interference free solutions exist for up to $K=3$ users. In this simple scenario, the solutions can even be expressed in closed form, as will be shown in the following.

Proposition 1: Assuming that all cross channels $\boldsymbol{H}_{k j}$, for $j \neq k$, are invertible, zero interference can be achieved with unit-norm beamformers $\boldsymbol{v}_{1}, \boldsymbol{v}_{2}, \boldsymbol{v}_{3}$, if and only if $\boldsymbol{v}_{1}$ is an eigenvector of the matrix $\boldsymbol{H}_{21}^{-1} \boldsymbol{H}_{23} \boldsymbol{H}_{13}^{-1} \boldsymbol{H}_{12} \boldsymbol{H}_{32}^{-1} \boldsymbol{H}_{31}$, and $\boldsymbol{v}_{2} \| \boldsymbol{H}_{32}^{-1} \boldsymbol{H}_{31} \boldsymbol{v}_{1}$ and $\boldsymbol{v}_{3} \| \boldsymbol{H}_{23}^{-1} \boldsymbol{H}_{21} \boldsymbol{v}_{1}$, where $\boldsymbol{a} \| \boldsymbol{b}$ is equivalent to $\boldsymbol{a}=\lambda \boldsymbol{b}, \lambda \in \mathbb{C}$.

Proof (Sketch): From the two conditions at the first receiver, $\boldsymbol{g}_{1}^{\mathrm{H}} \boldsymbol{H}_{12} \boldsymbol{v}_{2}=0$ and $\boldsymbol{g}_{1}^{\mathrm{H}} \boldsymbol{H}_{13} \boldsymbol{v}_{3}=0$, and from $\boldsymbol{g}_{1} \in \mathbb{C}^{2}$, it follows that $\boldsymbol{H}_{12} \boldsymbol{v}_{2} \| \boldsymbol{H}_{13} \boldsymbol{v}_{3}$. Similarly, $\boldsymbol{H}_{21} \boldsymbol{v}_{1} \| \boldsymbol{H}_{23} \boldsymbol{v}_{3}$ and $\boldsymbol{H}_{31} \boldsymbol{v}_{1} \| \boldsymbol{H}_{32} \boldsymbol{v}_{2}$ from the conditions at the second and third receiver. The proposition follows directly from eliminating $\boldsymbol{v}_{2}$ and $\boldsymbol{v}_{3}$ and solving the system of equations for $\boldsymbol{v}_{1}$.

Due to the Gaussian i.i.d. channel model, the matrices are invertible almost surely, so that there are exactly two sets of beamformers fulfilling the conditions almost surely. For each set of beamformers $\boldsymbol{v}_{1}, \boldsymbol{v}_{2}$, and $\boldsymbol{v}_{3}$, the receive filters $\boldsymbol{g}_{1}, \boldsymbol{g}_{2}$, and $\boldsymbol{g}_{3}$ immediately follow from the zero-interference conditions.

As discussed in the previous section, the aligned beamformers and receivers do not depend on the direct channels $\boldsymbol{H}_{11}$, $\boldsymbol{H}_{22}$, and $\boldsymbol{H}_{33}$. The optimal strategy at high SNR is therefore to compute both solutions and use the one that results in a higher value of $r$.

As a preliminary step to approximating the average rate offset, fix two arbitrary unit-norm vectors $\boldsymbol{v}_{1}$ and $\boldsymbol{g}_{1}$ and let $\boldsymbol{H}_{11}$ be a random matrix with unit-variance complex Gaussian i. i. d. elements.

Proposition 2: The random variable $x=\log \left|\boldsymbol{g}_{1}^{\mathrm{H}} \boldsymbol{H}_{11} \boldsymbol{v}_{1}\right|^{2}$ has the probability density function (pdf)

$$
f_{x}(x)=\mathrm{e}^{x} \mathrm{e}^{-\mathrm{e}^{x}}
$$

which is a reversed Gumbel distribution. The mean of $x$ is $-\gamma$ (where $\gamma$ is the Euler-Mascheroni constant) and the variance is $\pi^{2} / 6$.

Proof (Sketch): The distribution of $\boldsymbol{g}_{1}^{\mathrm{H}} \boldsymbol{H}_{11} \boldsymbol{v}_{1}$ is complex Gaussian with unit variance; it is Gaussian since it is a weighted sum of Gaussians, the proof for unit variance is omitted, but straightforward. The random variable $z=\left|\boldsymbol{g}_{1}^{\mathrm{H}} \boldsymbol{H}_{11} \boldsymbol{v}_{1}\right|^{2}$ is the sum of the squares of the real and imaginary part of $\boldsymbol{g}_{1}^{\mathrm{H}} \boldsymbol{H}_{11} \boldsymbol{v}_{1}$, which are both Gaussian with variance $\frac{1}{2}$. Therefore, $2 z$ has a Chi-square distribution with two degrees of freedom [4], and $z$ has the pdf

$$
f_{z}(z)=\mathrm{e}^{-z} \quad \text { if } \quad z \geq 0, \quad \text { zero otherwise. }
$$

By transformation of the random variable, we find that $x=$ $\log z$ has the preceding pdf. For the mean and variance, cf. [5, Section 9.3].

We would now like to approximate the average rate offset over channel realizations $\bar{r}$, and thus the average high SNR performance, for the following two strategies:

Random Alignment (RA): Compute the two aligned solutions and randomly decide which one to use.

Max-Alignment (MA): Compute the two aligned solutions and use the one with the higher rate offset $r$.

Proposition 3: For RA $\bar{r}$ is given by $\bar{r}_{\mathrm{RA}}=-3 \gamma \approx$ -1.732 .

Proof: $r$ is the sum of three independent random variables, each of which has the pdf of the variable $x$ in Proposition 2, cf. (5). Therefore the mean is the sum of the three means.

Determining $\bar{r}$ for MA is far more difficult. We therefore make the following simplifying assumptions, in order to obtain a useful approximation:

1) The distribution of a sum of three independent random variables, each of which has the pdf of the variable $x$ in Proposition 2, is approximately Gaussian with mean $-3 \gamma$ and variance $\pi^{2} / 2$.

2) Instead of choosing the better of two beamformer/receiver sets for a given channel realization, we consider a similar random experiment in which we compare two independent channel realizations for a given beamformer/receiver set. For large systems the two experiments are equivalent, cf. Section V.

With these approximations, the mean $\bar{r}_{\mathrm{MA}}$ is simply the expected value of the maximum of two realizations of a Gaussian random variable with mean $-3 \gamma$ and variance $\pi^{2} / 2$. This can be explicitly calculated by determining the pdf of the maximum and solving the integral for the expectation. The result of the approximation for the average rate offset with the optimal MA strategy is $\bar{r}_{\mathrm{MA}}=-3 \gamma+\sqrt{\pi / 2} \approx-0.478$, where the details of the derivation are omitted due to space constraints.

For the numerical evaluation of our approximation, we averaged the sum rate performance of the RA and MA strategies over 1000 random channel realizations and plotted the simulated performance alongside the derived high-SNR approximations in Fig. 2. These results shows that the approximation for $\bar{r}_{\mathrm{MA}}$ is very accurate.

\section{LARGE SySTEM APPROXIMATION}

In this section, we perform a similar analysis for systems with a large number $N$ of antennas and $K=2 N-1$ users. As discussed in Section III, the number of equations equals the number of variables in this case and we assume that there is a finite number of aligned solutions. For the analysis, it is 


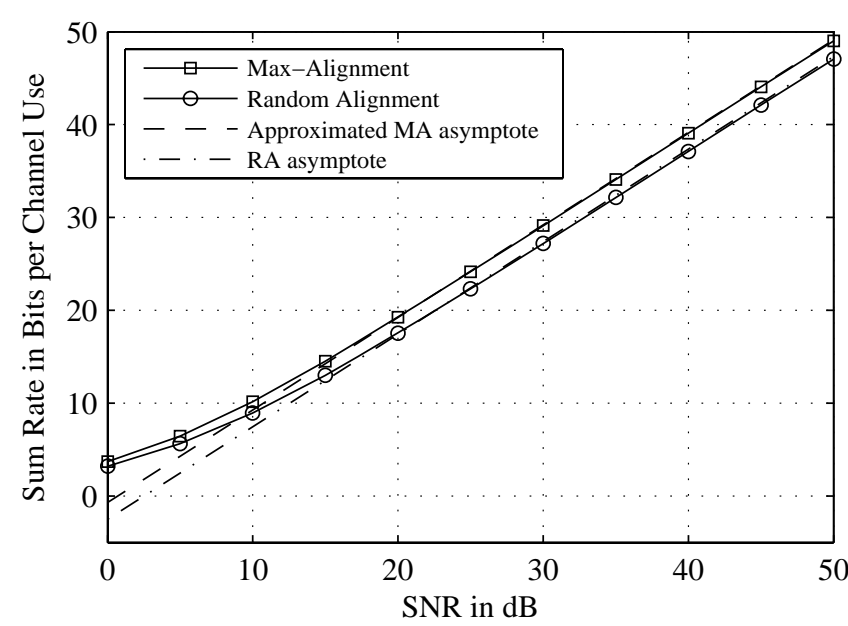

Fig. 2. Analytical and simulated performance results for $N=2$.

crucial to know how many solutions we can choose from; this is, however, unknown for $N>2$.

An upper bound is given by Bézout's Theorem [2], [3], [6], which states that the number of solutions to a set of multivariate polynomial equations is at most the product of the degrees of the individual equations. Following the discussion in Section III, the product of the degrees is $2^{2 K^{2}}=2^{2(2 N-1)^{2}}$ in our case. The expression varies when the interference alignment conditions are stated in a different way, but the upper bound always grows as $\mathrm{O}\left(c^{N^{2}+\alpha N}\right)$ for $N \rightarrow \infty$, with some constants $c>1$ and $\alpha \in \mathbb{R}$.

Clearly, the number of solutions might be extremely high for $N>2$ and evaluating $r$ for each solution could be computationally infeasible. Instead, we assume that we have some method for computing different aligned solutions from the cross-channel coefficients, where computing more solutions incurs an additional computational cost. An example for such a method is to run an iterative algorithm, such as the 'Minimum Leakage' algorithm of [7], with different initializations.

Max-of-L Alignment (MLA): Compute $L$ different aligned solutions and use the one with the largest rate offset $r$.

In the following, we approximate $\bar{r}$ with the MLA strategy with different assumptions concerning the relation between $L$ and $N$. For this analysis we make the simplifying assumption that the $L$ aligned strategies are independent sets of random isotropically distributed unit-norm beamforming and receive filter vectors. ${ }^{1}$

First consider a preliminary random experiment with unit norm vectors $\boldsymbol{v}_{1}$ and $\boldsymbol{g}_{1}$ that are isotropically distributed on the unit sphere, and the matrix $\boldsymbol{H}_{11}$, which has unit-variance complex Gaussian i.i.d. elements. In a large system, the singular values of $\boldsymbol{H}_{11}$ are deterministic [8]. Furthermore, the matrix containing the left and right singular bases are unitary, so that when they are multiplied with any unit norm vector,

\footnotetext{
${ }^{1}$ This assumption leads to an upper bound on the rate offset since correlation generally reduces the maximum value.
}

the resulting unit norm vector is also isotropically distributed on the unit sphere. Therefore, for a given realization $\boldsymbol{H}_{11}$, the distribution of $\boldsymbol{g}_{1}^{\mathrm{H}} \boldsymbol{H}_{11} \boldsymbol{v}_{1}$ over random $\boldsymbol{v}_{1}$ and $\boldsymbol{g}_{1}$ is the same as the distribution of $\boldsymbol{g}_{1}^{\mathrm{H}} \boldsymbol{H}_{11} \boldsymbol{v}_{1}$ when $\boldsymbol{v}_{1}$ and $\boldsymbol{g}_{1}$ are given realizations, and $\boldsymbol{H}_{11}$ is random.

With this insight, and from the discussion in Section IV, we obtain the distribution of one summand of $r$, cf. (6). Due to the large number of summands in (5), the distribution of $r$ is approximately Gaussian and has mean $-(2 N-1) \gamma$ and variance $(2 N-1) \pi^{2} / 6$.

Proposition 4 (cf. [5, Section 9.3]): For large L, the maximum out of $L$ realizations of a Gaussian random variable with mean $-(2 N-1) \gamma$ and variance $(2 N-1) \pi^{2} / 6$ follows a scaled and shifted Gumbel distribution, the mean of which is $-(2 N-1) \gamma+\pi \sqrt{(2 N-1) / 6}(\ell+\gamma / \ell)$, where $\ell=Q^{-1}(1 / L)$ and $Q^{-1}(\cdot)$ is the inverse of the Q-function.

Assuming that the Gaussian approximation is accurate for large $N$, we can therefore use this as an approximation for $\bar{r}_{\text {MLA }}$. Note, however, that $\ell=\mathrm{O}(\sqrt{\log L})$ as $L \rightarrow \infty$ [5]. Consequently, for $L=c^{N^{2}+\alpha N}$, i. e., if the upper bound on the number of solutions provided by Bézout's Theorem is tight and we are able to try out all possible solutions, $\ell$ is $\mathrm{O}(N)$ for $N \rightarrow \infty$ and the approximated mean $\bar{r}_{\text {MLA }}$ is $\mathrm{O}\left(N^{\frac{3}{2}}\right)$. This is a higher growth rate than that of a simple upper bound in which the interference channels are set to zero, which yields $\mathrm{O}(N \log N)$. This indicates that when taking the maximum out of so many realizations, we are in a regime so far right on the tail of the pdf that the Gaussian assumption no longer holds, so that our approximations do not provide a meaningful performance characterization.

Nonetheless, when $L$ increases more slowly (e.g., exponentially) with $N$, the Gaussian approximation is reasonable and leads to accurate performance estimates of finite-size systems, as indicated by the numerical results in Section VII. We also note that for any polynomial relationship $L=N^{m}$ with $m \geq 1$, the analysis shows that $\bar{r}_{\text {MLA }} \rightarrow-\infty$ for $N \rightarrow \infty$, i. e., the y-axis intercept of the asymptote of the average sum rate moves down, while the slope increases, as the system size grows. Note that this asymptotic rate offset is the same as that obtained by choosing a random aligned solution $(L=1)$. We will investigate the consequences of this effect in Section VIII.

\section{Achievable Rate Offset with $K=N$}

We would like to compare the previously discussed full alignment case to a scheme in which $K \leq N$ users are served. Assuming zero interference, the high-SNR slope is at most $s=N$, instead of $2 N-1$ for full alignment. When the number of active users does not exceed the number of antennas at each terminal, however, every set of beamformers $\boldsymbol{v}_{1}, \ldots, \boldsymbol{v}_{K}$ fulfills the zero-interference conditions (for a properly chosen set of receive filters $\boldsymbol{g}_{1}, \ldots, \boldsymbol{g}_{K}$ ).

Instead of searching for the globally optimal set of beamformers, we examine a suboptimal Successive Allocation (SA) strategy for $K \leq N$ users, similar to the scheme in [9]. We begin with the beamformer $\boldsymbol{v}_{1}$ and receiver $\boldsymbol{g}_{1}$, which are 
chosen to maximize the first user's individual rate offset, i. e., the first summand of $r$ in (5):

$$
\max _{\boldsymbol{v}_{1}, \boldsymbol{g}_{1}}\left|\boldsymbol{g}_{1}^{\mathrm{H}} \boldsymbol{H}_{11} \boldsymbol{v}_{1}\right|^{2} \quad \text { s.t.: } \quad\left\|\boldsymbol{v}_{1}\right\|_{2}^{2}=1 \quad \text { and } \quad\left\|\boldsymbol{g}_{1}\right\|_{2}^{2}=1 \text {. }
$$

The solution to this problem can be obtained by performing a singular value decomposition (SVD) of $\boldsymbol{H}_{11} ; \boldsymbol{v}_{1}$ and $\boldsymbol{g}_{1}$ are simply the principal right and left singular vectors, respectively. For the following users $k \in\{2, \ldots, K\}$, the beamformers and receive filters are chosen to maximize the respective individual rate offset, but subject to the condition that all interference terms involving the previously allocated users must be zero:

$$
\begin{aligned}
\max _{\boldsymbol{v}_{k}, \boldsymbol{g}_{k}}\left|\boldsymbol{g}_{k}^{\mathrm{H}} \boldsymbol{H}_{k k} \boldsymbol{v}_{k}\right|^{2} \quad \text { s. t.: } & \left\|\boldsymbol{v}_{k}\right\|_{2}^{2}=1 \quad \text { and }\left\|\boldsymbol{g}_{k}\right\|_{2}^{2}=1 \\
& \boldsymbol{g}_{k}^{\mathrm{H}} \boldsymbol{H}_{k j} \boldsymbol{v}_{j}=\boldsymbol{g}_{j}^{\mathrm{H}} \boldsymbol{H}_{j k} \boldsymbol{v}_{k}=0 \\
& \forall j \in\{1, \ldots, k-1\} .
\end{aligned}
$$

Here, the solution is found by performing the SVD of a projected version of $\boldsymbol{H}_{k k}$, where the projectors from the left and right are designed to ensure that the additional zeroforcing constraints are fulfilled.

Furthermore, it is possible to order the users, i. e., instead of beginning with user 1 , to begin with the user that has the highest maximum singular value of the direct channel, and then to continue with the user that has the highest maximum singular value of the appropriately projected direct channel, and so on.

Proposition 5: Regardless of how the users are ordered, as $N \rightarrow \infty$, the rate offset normalized with the system dimension $r / N$ of this scheme approaches $2 \log 2+\log (N !) / N$, i. e.,

$$
\lim _{N \rightarrow \infty} r / N-2 \log 2-\log (N !) / N=0 .
$$

Proof Sketch: In a large system, the singular values of $\boldsymbol{H}_{11}$ are deterministic and are governed by the MarčenkoPastur distribution; the maximum singular value approaches $2 \sqrt{N}$ (see, e.g., [8]). Therefore, the rate for the first user approaches $\log (4 N)$. The projected direct channel of the second user can be shown to have the same singular values as an $(N-1) \times(N-1)$ matrix with unit-variance complex Gaussian i. i.d. elements; its maximum singular value therefore approaches $2 \sqrt{N-1}$, the individual rate offset is $\log (4(N-1))$, etc. Now let $M=N-K=\epsilon N$ for some small $\epsilon>0$. The maximum singular value for the $N-M$ th user approaches $2 \sqrt{M}$ with rate offset $\log (4 M)$. Hence the sum rate offset excluding the last $M$ users approaches $r=2(N-M) \log 2+$ $\log (N !)-\log (M !)=2(1-\epsilon) N \log 2+\log (N !)-\log [(\epsilon N) !]$. Since $\epsilon$ can be arbitrarily small, we obtain (10).

The proposition implies that for a system with finite $K=N$ we can approximate $\bar{r}_{\mathrm{SA}} \approx 2 N \log 2+\log (N$ !), which grows as $\mathrm{O}(N \log N)$. We note that a similar argument can be used to estimate $r$ for a system with $K<N$. As can be seen in Section VII, this approximation is quite accurate, even for a moderately sized system.

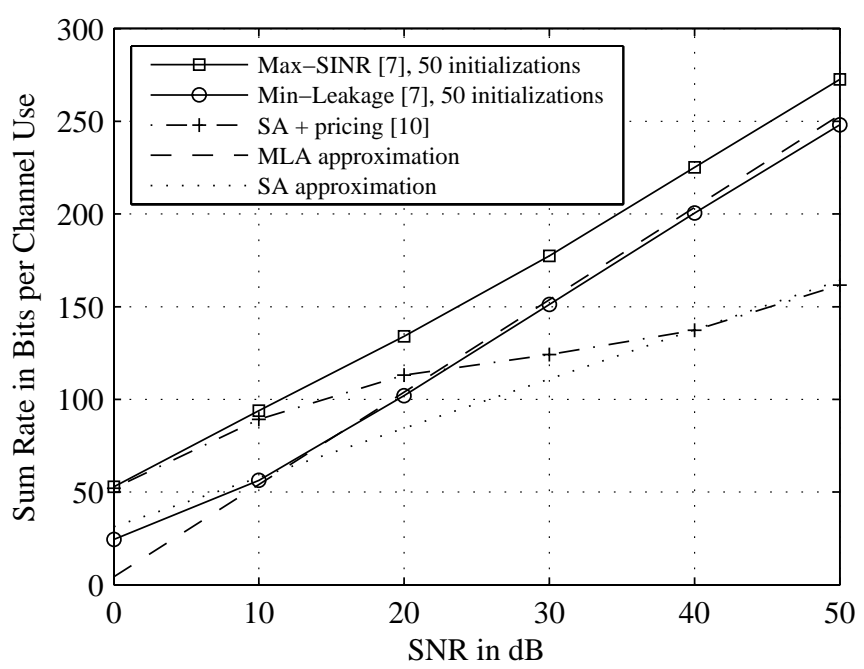

Fig. 3. Analytical and simulated performance results for $N=8$.

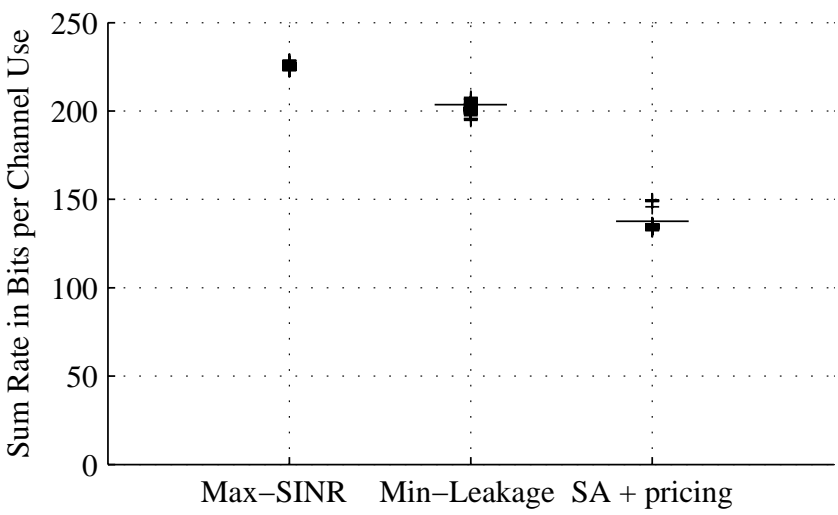

Fig. 4. Scatter plot showing the sum rate at $40 \mathrm{~dB}$ for 50 different channel realizations for the same scenario as in Fig. 3. The horizontal lines are the analytical approximations.

\section{NUMERICAL EVALUATION}

To evaluate the approximations, we simulated the MLA and SA strategies for 20 different channel realizations in a scenario with $N=8$ antennas per user. In order to obtain $L=50$ aligned solutions per channel realization for the MLA strategy, we let the 'Min-Leakage' algorithm from [7] run until convergence from 50 different random initial conditions. For comparison, we also let the 'Max-SINR' algorithm from [7] run until convergence from the same 50 initial conditions and chose the best result. The SA strategy was implemented with ordering as described in Section VI. To improve the performance of SA at low and moderate SNRs, we used the resulting strategy as an initial condition for the distributed optimization (pricing) algorithm in [10]. Note that the pricing algorithm does not deviate from an interference free strategy at high SNR. ${ }^{2}$

\footnotetext{
${ }^{2}$ The performance is measured in bits per channel use, which requires a scaling of the slope and offset expressions from the previous sections, where the natural logarithm is used.
} 


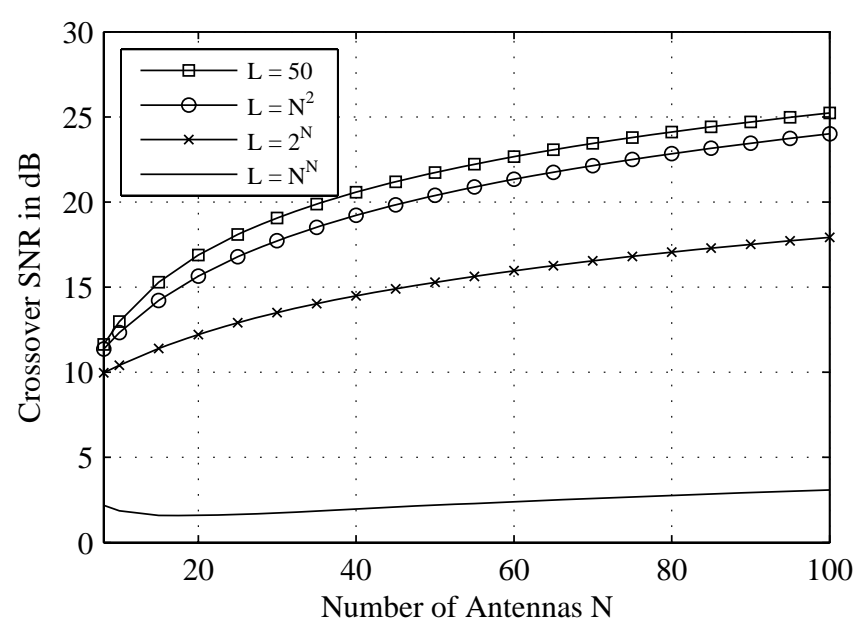

Fig. 5. Large system crossover SNR assuming different relationships for $L$ and $N$.

Fig. 3 shows that the analysis accurately predicts the simulated performance of the MLA and SA strategies at high SNRs. The 'Max-SINR' algorithm performs significantly better than the MLA and SA strategies due to the fact that the direct channels $\boldsymbol{H}_{k k}$ are taken into account in the iterative updates. Since the beamformers and receivers are no longer independent of the direct channels, our large system results do not apply to the 'Max-SINR' algorithm. For the channel model assumed here, it is not known whether the SA strategy can achieve a larger rate offset than the Max-SINR algorithm for larger $N$. We note, however, that as the variance of the crosschannel coefficients increases relative to the direct channel coefficients, the 'Max-SINR' curve moves closer to 'MinLeakage' curve.

Fig. 4 shows how the performance of the three schemes varies over 50 different random channel realizations in the same scenario at an SNR of $40 \mathrm{~dB}$. The two horizontal lines for the MLA and SA strategies mark the large system approximation for the sum rate.

\section{Asymptotic CRossover Point}

We are able to approximate the high-SNR asymptotes of an interference alignment based strategy (MLA) and a strategy that only serves $N$ users (SA). While the asymptotic slope for MLA is nearly twice as high as for SA, the offset for SA grows as $\mathrm{O}(N \log N)$ whereas for MLA it can even decrease with $N$ (e.g., when $L=N^{m}$ ). We characterize this trade-off by computing the SNR at which the two asymptotes cross:

$$
\log \left(\sigma_{\text {crossover }}^{-2}\right)=\frac{\bar{r}_{\mathrm{SA}}-\bar{r}_{\mathrm{MLA}}}{N-1} .
$$

For $L=N^{m}$, it is straightforward to see with the approximations in Propositions 4 and 5 that the crossover point moves to the right as $N \rightarrow \infty$, i. e., the SNR for which MLA outperforms SA becomes larger and larger. In Fig. 5, (11) is numerically evaluated using the large system results in Propositions 4 and 5 for different relationships between $L$ and
$N$. Interestingly, even for the super-exponential relationship $L=N^{N}$, the crossover point moves right as $N$ grows, albeit very slowly.

The results in Fig. 5 are based on large system approximations, and also the assumption that the $L$ aligned solutions give independent rate offsets. The latter leads to an upper bound on the rate offset, although it is not known how accurate this bound is for larger $N$. Nonetheless, the fact that the approximations accurately predict the performance of moderately sized systems indicates that they can be used to predict the performance of larger systems as well. This is quite useful, since simulating systems with larger $N$ quickly become very time consuming.

\section{Conclusion}

We have derived large system approximations for the behavior of the high-SNR asymptote of interference alignment strategies with a computational constraint, as well as for a scheme where the number of users is equal to the number of antennas and the beams are successively allocated. The approximations provide an accurate prediction of the highSNR performance of the associated schemes for moderately sized systems, which can be simulated with an acceptable computational effort. Assuming that the approximations also hold for larger systems, we show that the SNR at which the two asymptotes cross increases with the system size even if the number of different aligned solutions, which are sampled, grows exponentially with the system dimension.

\section{REFERENCES}

[1] V. R. Cadambe and S. A. Jafar, "Interference alignment and degrees of freedom of the $K$-user interference channel," IEEE Trans. Inf. Theory, vol. 54, no. 8, pp. 3425-3441, Aug. 2008.

[2] C. M. Yetis, T. Gou, S. A. Jafar, and A. H. Kayran, "Feasibility conditions for interference alignment," in Proc. IEEE Global Commun. Conf. (GLOBECOM), Honolulu, HI, Nov. 2009.

[3] —, "On feasibility of interference alignment in MIMO interference networks," arXiv.org, Nov. 2009. [Online]. Available: http://arxiv.org/abs/0911.4507v1

[4] A. Papoulis and S. U. Pillai, Probability, Random Variables and Stochastic Processes, 4th ed. New York, NY: McGraw-Hill, 2002.

[5] H. A. David, Order Statistics, 2nd ed. New York, NY: John Wiley \& Sons, Inc., 1981.

[6] W. Fulton, Introduction to Intersection Theory in Algebraic Geometry, 3rd ed., ser. Conference Board of the Mathematical Sciences, Regional Conference Series in Mathematics. Providence, RI: American Mathematical Society, 1996.

[7] K. Gomadam, V. R. Cadambe, and S. A. Jafar, "Approaching the capacity of wireless networks through distributed interference alignment," in Proc. IEEE Global Commun. Conf. (GLOBECOM), New Orleans, LA, Nov. 2008.

[8] M. J. M. Peacock, I. B. Collings, and M. L. Honig, Advances in Multiuser Detection, ser. Wiley Series in Telecommunications and Signal Processing. John Wiley \& Sons, 2009, vol. 1, ch. Performance with Random Signatures.

[9] C. Guthy, W. Utschick, and G. Dietl, "Low-complexity linear zeroforcing for the MIMO broadcast channel," IEEE J. Sel. Topics Signal Process., vol. 3, no. 6, pp. 1106-1117, Dec. 2009.

[10] C. Shi, D. A. Schmidt, R. A. Berry, M. L. Honig, and W. Utschick, "Distributed interference pricing for the MIMO interference channel," in Proc. IEEE Int. Conf. Commun., Dresden, Germany, Jun. 2009. 